\title{
Article
}

\section{Hole Hopping through Cytochrome P450}

Mette L.H. Sørensen, Brian C. Sanders, L. Perry Hicks, Maria H. Rasmussen, Andreas

L. Vishart, Jacob Kongsted, Jay R. Winkler, Harry B. Gray, and Thorsten Hansen

J. Phys. Chem. B, Just Accepted Manuscript • DOI: 10.1021/acs.jpcb.9b09414 • Publication Date (Web): 16 Mar 2020

Downloaded from pubs.acs.org on March 16, 2020

\section{Just Accepted}

"Just Accepted" manuscripts have been peer-reviewed and accepted for publication. They are posted online prior to technical editing, formatting for publication and author proofing. The American Chemical Society provides "Just Accepted" as a service to the research community to expedite the dissemination of scientific material as soon as possible after acceptance. "Just Accepted" manuscripts appear in full in PDF format accompanied by an HTML abstract. "Just Accepted" manuscripts have been fully peer reviewed, but should not be considered the official version of record. They are citable by the Digital Object Identifier (DOI®). "Just Accepted" is an optional service offered to authors. Therefore, the "Just Accepted" Web site may not include all articles that will be published in the journal. After a manuscript is technically edited and formatted, it will be removed from the "Just Accepted" Web site and published as an ASAP article. Note that technical editing may introduce minor changes to the manuscript text and/or graphics which could affect content, and all legal disclaimers and ethical guidelines that apply to the journal pertain. ACS cannot be held responsible for errors or consequences arising from the use of information contained in these "Just Accepted" manuscripts. 


\title{
Hole Hopping through Cytochrome P450
}

\author{
Mette L. H. Sørensen, ${ }^{\dagger} \|$ Brian C. Sanders, ${ }^{\ddagger}, \|$ L. Perry Hicks, $₫$ Maria H. \\ Rasmussen, ${ }^{\dagger}$ Andreas L. Vishart, ${ }^{\dagger}$ Jacob Kongsted, ${ }^{\S}$ Jay R. Winkler, $₫$ Harry B. \\ Gray, and Thorsten Hansen*,† \\ $\dagger$ Department of Chemistry, University of Copenhagen, Universitetsparken 5, DK 2100 \\ Copenhagen Ø, Denmark \\ $\ddagger$ Environmental Sciences Division, Oak Ridge National Laboratory, 1 Bethel Valley Road, \\ Oak Ridge, TN 37831, USA \\ ФDivision of Chemistry and Chemical Engineering, California Institute of Technology, 1200 \\ E California Blvd, Pasadena, CA 91125, USA \\ $\S$ Department of Physics, Chemistry, and Pharmacy, University of Southern Denmark, \\ Campusvej 55, DK 5230 Odense M, Denmark \\ \| These authors contribued equally to this work. \\ E-mail: thorsten@chem.ku.dk
}

Phone: +45 22295728 


\begin{abstract}
High-potential iron-oxo species are intermediates in the catalytic cycles of oxygenase enzymes. They can cause heme degradation via irreversible oxidation of nearby amino acids. We have proposed that there are protective mechanisms in which hole hopping from oxidized hemes through tryptophan/tyrosine chains generates a surface-exposed amino-acid oxidant that could be rapidly disarmed by reaction with cellular reductants. In investigations of cytochrome $\mathrm{P} 450_{B M 3}$, we identified Trp96 as a critical residue that could play such a protective role. This Trp is cation- $\pi$ paired with Arg398 in $81 \%$ of mammalian P450s. Here we report on the effect of the Trp/Arg cation- $\pi$ interaction on Trp96 formal potentials as well as on electronic coupling strengths between Trp96 and the heme both for wild type cytochrome P450 and selected mutants. Mutation of Arg398 to His, which decreases the Trp96 formal potential, increases Trp-heme electronic coupling; but, surprisingly, the rate of phototriggered electron transfer from a Ru-sensitizer (through Trp96) to the P450 $B M 3$ heme was unaffected by the Arg398His mutation. We conclude that Trp96 has moved away from Arg398, suggesting that the protective mechanism for P450s with this Trp-Arg pair is conformationally gated.
\end{abstract}

\title{
Introduction
}

Cytochrome P450s, found in nearly all living systems, are monooxygenase enzymes that play critical roles in the production of hormones and the metabolism of xenobiotics. ${ }^{1}$ The active site of $\mathrm{P} 450 \mathrm{~s}$ features a thiolate-ligated heme $\mathrm{B}$ capable of reacting with $\mathrm{O}_{2}, 2 \mathrm{H}^{+}$, and $2 \mathrm{e}^{-}$to form a powerful oxidant, an $\mathrm{Fe}(\mathrm{IV})$-oxo porphyrin cation radical $\left(\mathrm{Por}+{ }^{\bullet}\right.$ ) known as Compound I (Cpd I). ${ }^{2}$ The ability of P450s to activate and hydroxylate strong C-H bonds with both regio- and stereospecificity has made these enzymes the poster childs of both human health and biotechnology research programs. ${ }^{3-5}$ The catalytic mechanisms of P450s, which have been studied extensively, involve the formation of Cpd I, followed by H-atom abstraction from the substrate, forming an $\mathrm{Fe}(\mathrm{IV})-\mathrm{OH}$ porphyrin called Compound II (Cpd 
II) and a substrate radical $\left(\mathrm{Sub}^{\bullet}\right)$. The next step, a radical rebound of $\mathrm{HO} \bullet$ from $\mathrm{Cpd}$ II to $\mathrm{Sub}^{\bullet}$, affords the hydroxylated product. ${ }^{6}$ Although we have a good understanding of the basic steps in the catalytic cycle, we know much less about the role of conformational dynamics in substrate binding/release and control of electron flow through the enzyme, two of the factors that determine the efficiency of enzymatic function (coupled turnover).

As the functions of cytochrome $\mathrm{P} 450$ require the generation of a potent oxidant, Cpd I, there must be, we think, protective mechanisms against self-inflicted oxidative damage in the event of uncoupled turnover. One possible protective mechanism is based on the ejection of electron holes from Cpd I to the protein surface through redox active amino acids. Such a mechanism could be tested by variation in the local environment of individual amino acids, or by mutation of amino acids in the hole hopping chain, as these would affect the electronic coupling and reduction potentials of participating residues.

Experimental work involving LC-MS/MS analysis of cytochrome c peroxidase (Ccp1) under oxidative stress conditions, and photochemical oxidation of ruthenium $(\mathrm{Ru})$-modified P450 key amino acids involved in electron hole transport through these oxidative enzymes. ${ }^{7-9}$ In a theoretical study, Beratan et al. found that electron hole transfer in P450S $\mathrm{SCC}_{\mathrm{SC}}$ (Cyp11a1), $\mathrm{P} 450_{\mathrm{BM} 3}$, and $\mathrm{Ccp} 1$ is efficient through pathways identified in experimental work and protein structure analysis. ${ }^{10,11}$

Work by Ener et al. involving the photooxidation of Ru-labeled P450 $0_{\mathrm{BM} 3}\left(\mathrm{RuP} 450_{\mathrm{BM} 3}\right)$ demonstrated that Trp96 is critical for hole injection from the surface photosensitizer to the heme, generating Cpd II. ${ }^{7,12}$ The photochemical cycle, shown in Figure 1, is reversible and occurs thousands of times without enzyme degradation. Notably, the mutation of Trp96 to histidine (His) precludes heme oxidation indicating that Trp96 is an essential component in the photochemical process. Moreover, Trp96 is a prime candidate for electron hole ejection in the reverse direction, from Cpd I to the enzyme surface, owing to its proximity to the heme $(7.0 \AA$, heme edge to tryptophan (Trp)-N). 


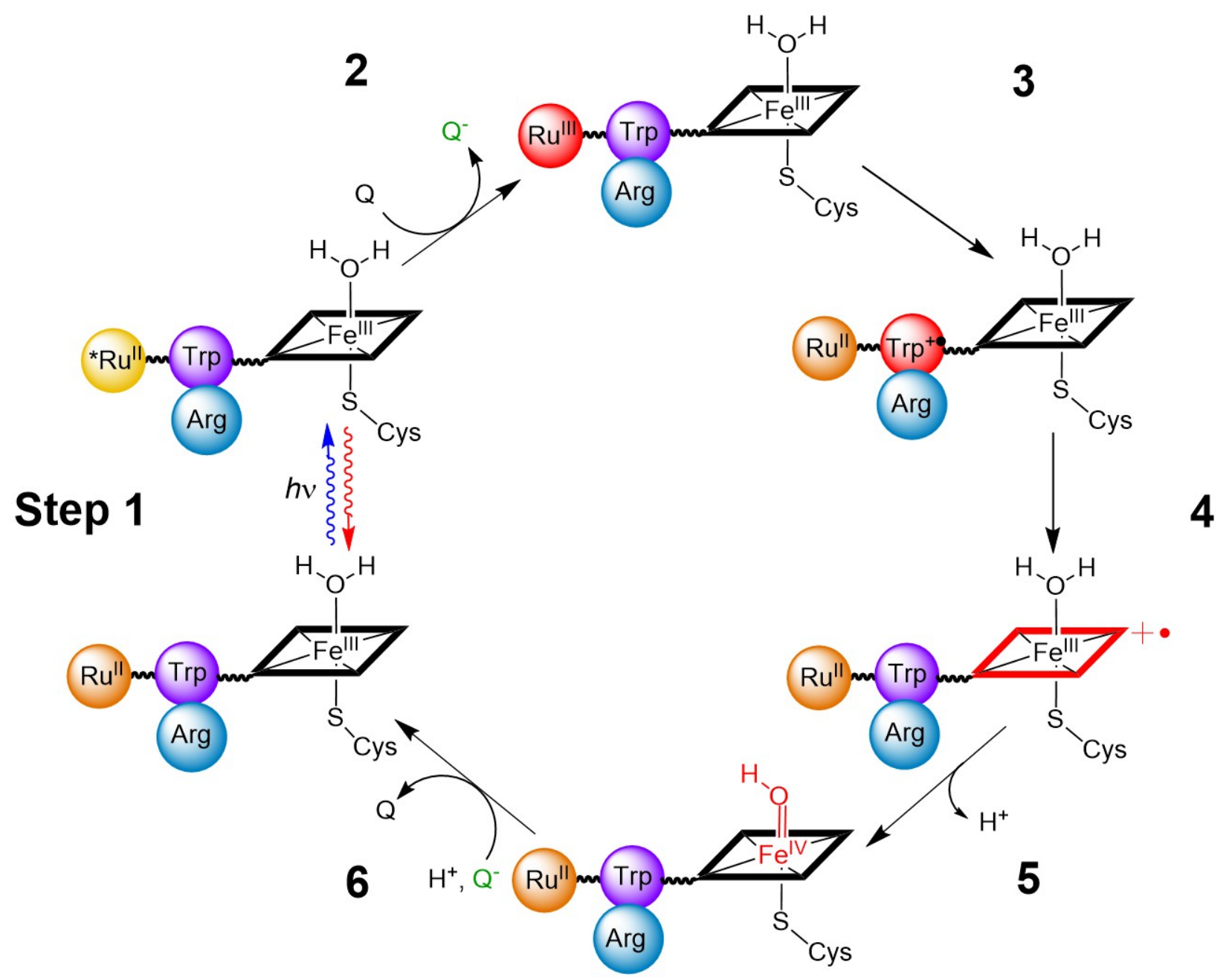

FIGURE. 1: RuP450 $0_{\text {BM3 }}$ photooxidation cycle. Species shown in red represent oxidized intermediates in the reversible cycle. As drawn, the Trp96 Arg398 cation- $\pi$ interaction would be in close contact as is observed in the crystal structures PDB: 3NPL and 3R1A. The photooxidation cycle begins with a laser pulse that excites the covalently attached $\mathrm{Ru}(\mathrm{II})$ photosensitizer, Step 1. This excited species is then quenched oxidatively $(\mathrm{Q}=$ Quencher $=$ $\left[\mathrm{Ru}\left(\mathrm{NH}_{3}\right)_{6}\right] \mathrm{Cl}_{3}$, generating $\mathrm{Ru}(\mathrm{III})$-photosensitizer, Step 2, and triggering the oxidation of Trp, porphyrin, and iron, forming Cpd II, Steps 3-5. The last step involves the reaction of reduced quencher (Q-) with Cpd II and returns the system to its initial state, Step 6. 

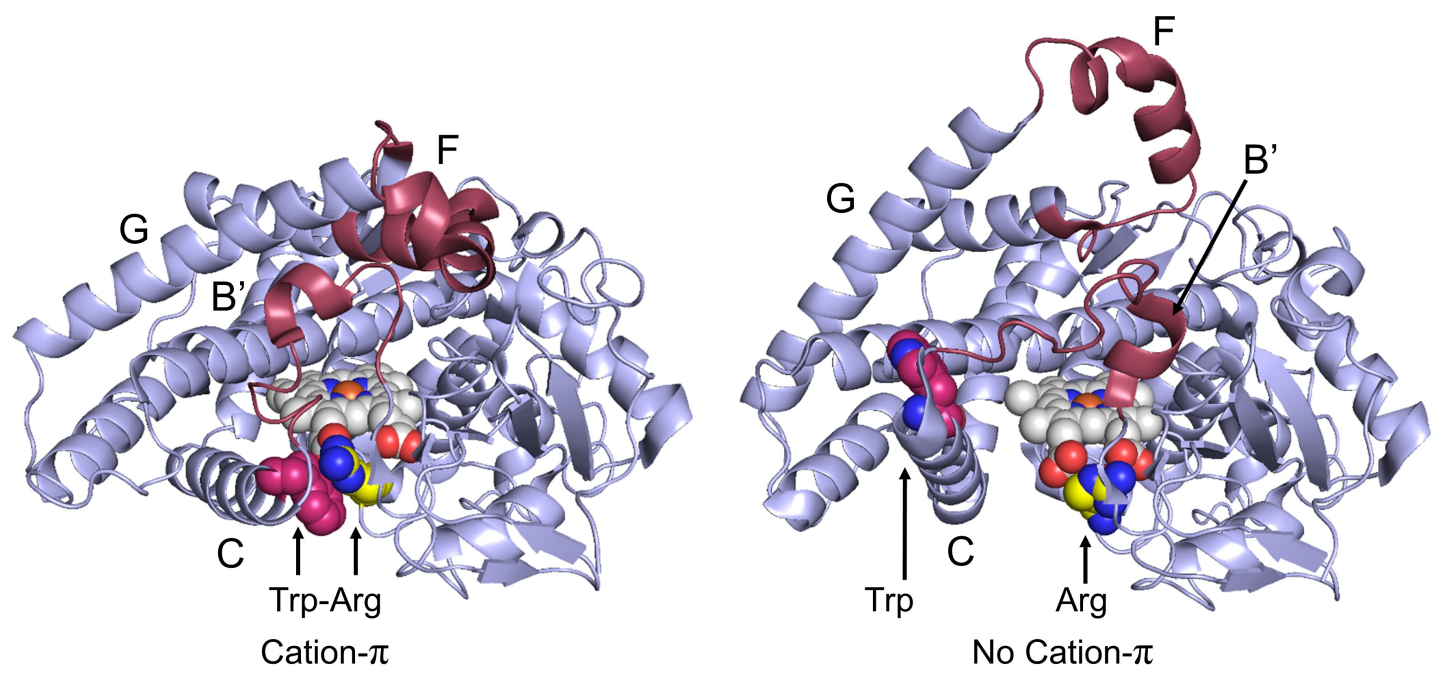

FIGURE. 2: Conformational changes between the open and closed conformations of P4502b4 from 3R1A (left) and 1PO5 (right). Region with the most change is colored maroon, heme (white and blue), Fe (orange), Arginine (yellow and blue), Tryptophan (magenta and blue). Notably, portions of the $\mathrm{C}$ helix containing Trp120 (Trp96 is $\mathrm{P} 450_{B M 3}$ numbering) move, dramatically increasing the Trp-Arg distance. Helices with significant conformational change are labelled.

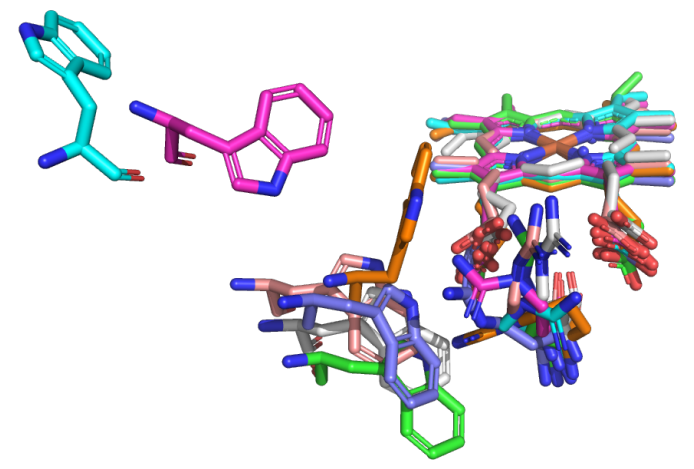

FIGURE. 3: Geometry of: (blue) 1PO5, (magenta) 2BDM, (orange) 3R1B, (purple) 3R1A, (pink) 3G5N, (white) 3G93, and (green) 1DT6. 
In $\mathrm{P} 450_{\mathrm{BM} 3}$, Trp96 is engaged in a cation- $\pi$ interaction with an arginine residue (Arg398). Polypeptide sequence alignments suggests that a Trp/Arg pair at this location is present in $81 \%$ of mammalian P450s. But, bacterial enzymes often have a His/His motif in these positions interacting with the heme propionate. Among the many P450 enzymes having this structurally analogous Trp-Arg pair, noteworthy examples are the mammalian Cyp3a4 and Cyp2b4 (P450 2b4). Cation- $\pi$ contacts are generally recognized motifs that stabilize protein structures. ${ }^{13}$ However, there has been little discussion in the literature regarding the cation- $\pi$ interaction acting as a non-covalent modulator of reduction potentials of aromatic amino acids. An electrochemical study involving a designed 3-helix bundle protein exhibited an increased reduction potential by $0.35 \mathrm{~V}$ vs. NHE as compared to free $N$-acetylL-tryptophanamide. ${ }^{14}$ The increased potential was attributed to a Lys-Trp cation- $\pi$ interaction in the folded protein. ${ }^{15}$ Additionally, aryl-sulfur interactions have gained attention as non-covalent contacts that affect reduction potentials. ${ }^{16,17}$ Moreover, a recent computational study suggests that a transient and flexible cation- $\pi$ interaction between Tyr154 and Lys131 in the Bacteriophage- $\lambda$ Exonuclease is an essential component of the catalytic cycle. ${ }^{18}$ It follows that conformational dynamics in enzymes likely cause reduction potential fluctuations in redox active amino acids. Thereby, the electron transfer pathways, which play critical roles in function and protection, are engaged and/or altered through conformational changes.

The conformational dynamics of P450 enzymes have been linked to substrate access, product exit, and the diversity of tolerated substrates. An excellent example of conformational flexibility is seen in mammalian $\mathrm{P} 450_{2 \mathrm{~b} 4},{ }^{19}$ the open and closed conformations of this enzyme are shown in Figure 2. In the open conformation (PDB 1PO5), a large cleft is formed by movement of regions including helices $\mathrm{F}$ to $\mathrm{G}$ and $\mathrm{B}$ ' to $\mathrm{C}$, allowing access from the enzyme surface to the heme active site. Importantly, helix C includes Trp120 (2b4 numbering), a residue analogous to Trp96 in $\mathrm{P} 450_{\mathrm{BM} 3}$. A large conformational change in helix $\mathrm{C}$ results in displacement of Trp120 from its cation- $\pi$ contact with Arg434 (PDB 3R1A) in the closed conformation. This Trp residue is displaced by approximately $14.7 \AA$ from the closed to 


\section{Methods}

\section{DFT Calculations}

All calculations were performed using the Gaussian16 program. ${ }^{24}$ We use the M06 functional, which is built for non-covalent interactions - such as the cation- $\pi$ interaction between Trp and Arg. ${ }^{25}$ The basis set $6-31 \mathrm{G}++(\mathrm{d}, \mathrm{p})$ was used for calculating absolute reduction potentials. For the electronic coupling calculations, we had to use the smaller basis set 6-31G(d,p), since the extraction of electronic coupling elements rely on partitioning schemes, which become ambiguous for very large basis sets. ${ }^{26-29}$ The protein surrounding a cluster was modeled using the polarizable continuum model (PCM) with a dielectric constant of 3.5 corresponding to the 
low shielding of the protein environment. ${ }^{30,31}$ The absolute reduction potential is calculated as the energy difference between the cationic $\operatorname{Trp}^{\bullet+}$ and the neutral Trp:

$$
\Delta E=E_{t r p} \bullet-E_{t r p}
$$

Entropy contributions are ignored in this work.

\section{Protein Structures}

The geometries used were downloaded directly from the P450 PDB files using Pymol. Appropriate hydrogen atoms where added and removed. The heavy atoms were frozen and an optimization of the hydrogen atoms was performed. In order to study the effect of the cationic Arg in the wild types, this site was mutated to a neutral His in some structures. Mutations were performed using the Pymol software package, which suggests mutant geometries based on PDB data. ${ }^{32}$

\section{Electronic Coupling Elements}

The electronic coupling element between donor and acceptor states is a key property for describing electron transfer. To estimate the electronic coupling, we use two simple methods from the literature. The first method, fragment charge difference (FCD), uses a simple two state model, while the second method, Projection-operator diabatization (POD), is based on a block diagonalization of the Fock/Kohn-Sham matrix.

\section{Fragment Charge Difference (FCD)}

The FCD method uses the charge difference operator to construct charge-localized orbitals. ${ }^{33}$ So, the adiabatic molecular orbitals (MOs) are rotated into the diabatic orbitals involved in an ET reaction. Therefore, new diabatic orbitals are constructed from the two chargelocalized adiabatic MOs and then optimized for the largest charge separation, which will 
correspond to the diabatic orbitals for the ET reaction. ${ }^{34}$ The two adiabatic MOs must be comparable to the diabatic MOs involved in the ET reaction.

The Mulliken population analysis is used to calculate the charge of the system for the adiabatic donor molecular orbital (D-MO) and the acceptor molecular orbital (A-MO). ${ }^{35}$ Koopmans' theorem must be assumed to be valid when only two adiabatic MOs are used.

Furthermore, it is assumed that the ET reaction is a one-electron transfer. The electronic coupling, $V_{D A}$, between the donor and acceptor diabatic states can be calculated with the FCD method as:

$$
V_{D A}=\frac{\left|\Delta q_{12}\right|\left(E_{A}-E_{D}\right)}{\sqrt{(\Delta q(D)-\Delta q(A))^{2}+4\left(\Delta q_{12}\right)^{2}}}
$$

where $\Delta q_{12}$ is the transition charge difference, $E_{A}$ is the Kohn-Sham MO energy for the A-MO, $E_{D}$ is the Kohn-Sham MO energy for the D-MO, $\Delta q(F)$ is the charge difference before and after the ET reaction at fragment $F$.

The charge difference at fragment $F$ is expressed by:

$$
\Delta q(F)=\sum_{k \in F} C_{D, k} \sum_{l}^{M} C_{D, l} S_{k l}-\sum_{k \in F} C_{A, k} \sum_{l}^{M} C_{A, l} S_{k l}
$$

where $C_{D, k}$ is an atomic orbital (AO) coefficient for the D-MO, $C_{A, k}$ is an $\mathrm{AO}$ coefficient for the A-MO, $k \in F$ means an AO localized on Fragment $F$ and $S_{k l}$ is an element in the overlap matrix of the $\mathrm{AO}, \mathrm{M}$ is the total number of basis functions ( $\mathrm{AO}$ ) in the basis set.

The transition charge difference is expressed as:

$$
\begin{aligned}
\Delta q_{12} & =\frac{1}{2}\left(\sum_{k \in A} C_{D, k} \sum_{l}^{M} C_{A, l} S_{k l}+\sum_{k \in A} C_{A, k} \sum_{l}^{M} C_{D, l} S_{k l}\right) \\
& -\frac{1}{2}\left(\sum_{k \in D} C_{D, k} \sum_{l}^{M} C_{A, l} S_{k l}+\sum_{k \in A} C_{A, k} \sum_{l}^{M} C_{D, l} S_{k l}\right)
\end{aligned}
$$




\section{Projection-Operator Diabatization (POD)}

POD, another simple and cost-efficient method of calculating electronic coupling elements between orbitals, was proposed by Kondov et al. ${ }^{36}$ For electron hole transfer on simple organic dimer systems, it has been shown to give errors similar to constrained DFT (CDFT) (POD and CDFT couplings deviate $9.3 \%$ and $5.3 \%$ from the correct results of the HAB11 test set. ${ }^{37}$ In the POD method the electronic coupling element between two orbitals, $\phi_{i}^{D}$ and $\phi_{j}^{A}$, localized on donor and acceptor, respectively, is given by the corresponding matrix element of the Fock operator, $F_{i j}$ (in the DFT formalism, the Kohn-Sham operator). The localized orbitals are then defined in terms of the Fock matrix expressed in the AO basis, $\mathbf{F}^{\mathbf{A O}}$. Since the AO basis functions are localized on the nuclei they can be partitioned according to donor and acceptor, respectively. The first step in the POD method is to utilize the localization of atomic orbitals to order the Fock matrix according to donor and acceptor orbitals. To work in an orthonormal basis, the AOs are Löwdin transformed and the Fock matrix in this orthonormal basis is:

$$
\tilde{\boldsymbol{F}}^{A O}=S^{-1 / 2} \boldsymbol{F}^{A O} S^{-1 / 2}=\left[\begin{array}{cc}
\tilde{\boldsymbol{F}}_{D D} & \tilde{\boldsymbol{F}}_{D A} \\
\tilde{\boldsymbol{F}}_{A D} & \tilde{\boldsymbol{F}}_{A A}
\end{array}\right]
$$

where $S$ is the overlap matrix for the AO basis. The Löwdin transformation is used since it creates the orthonormal basis closest to the original basis in a least squares sense. ${ }^{38,39}$ The donor and acceptor orbitals are now defined as the orbitals that diagonalize the donor and acceptor block, respectively:

$$
\begin{aligned}
& \varepsilon_{i}^{D}=\boldsymbol{\phi}_{\boldsymbol{i}}^{\boldsymbol{D}, \dagger} \tilde{\boldsymbol{F}}_{\boldsymbol{D} \boldsymbol{D}} \boldsymbol{\phi}_{\boldsymbol{i}}^{\boldsymbol{D}} \\
& \varepsilon_{j}^{A}=\boldsymbol{\phi}_{\boldsymbol{j}}^{\boldsymbol{A}, \dagger} \tilde{\boldsymbol{F}}_{\boldsymbol{A} \boldsymbol{A}} \boldsymbol{\phi}_{\boldsymbol{j}}^{\boldsymbol{A}}
\end{aligned}
$$


The Fock matrix can now be expressed in this localized and orthonomal basis:

$$
\left[\begin{array}{cc}
\varepsilon^{D} & F_{l o c}^{D A} \\
F_{l o c}^{A D} & \varepsilon^{A}
\end{array}\right]
$$

where $\varepsilon^{D}$ and $\varepsilon^{A}$ are diagonal matrices containing the donor and acceptor orbital energies respectively while the matrices on the off-diagonal, $\boldsymbol{F}_{\boldsymbol{l o c}}^{\boldsymbol{D A}}$ and $\boldsymbol{F}_{\boldsymbol{l o c}}^{\boldsymbol{A D}}$ are found as

$$
\begin{aligned}
& F_{l o c}^{D A}=\phi^{D, \dagger} \tilde{\boldsymbol{F}}_{D A} \phi^{A} \\
& \boldsymbol{F}_{l o c}^{A D}=\phi^{A, \dagger} \tilde{\boldsymbol{F}}_{A D} \phi^{D}
\end{aligned}
$$

and contain the electronic coupling elements between orbitals localized on donor and acceptor fragment, respectively.

\section{Experimental}

\section{Protein Expression, Labeling, and Characterization}

The wild type $\mathrm{P} 450_{\mathrm{BM} 3}$ heme domain was cloned into the pEt-22b(+) vector. A triple mutant protein $(\mathrm{C} 62 \mathrm{~A} / \mathrm{C} 156 \mathrm{~S} / \mathrm{K} 97 \mathrm{C})$ was prepared to remove two cysteines and introduce a new cysteine for conjugation with the photosensitizer. A quadruple mutant was prepared having the additional R398H mutation. All primers were purchased from Eurofins Genomics. In order to express the enzymes, a $25 \mathrm{~mL}$ stock culture of BL21(DE3) E. coli containing each mutant plasmid was grown shaken at $180 \mathrm{rpm}$ overnight at $37^{\circ} \mathrm{C}$ in $\mathrm{LB}$ broth. This stock culture was used to innoculate $2 \mathrm{~L}$ of TB broth in a $6 \mathrm{~L}$ flask and grown to an OD of 0.6 at $37^{\circ} \mathrm{C}$, shaken at $160 \mathrm{rpm}$. At this point, the cultures were charged with $0.5 \mathrm{mM}$ of 5-aminolevulinic acid and $1.0 \mathrm{mM}$ IPTG and the temperature was lowered to $30{ }^{\circ} \mathrm{C}$ for $16 \mathrm{~h}$, shaken at $110 \mathrm{rpm}$. Cell pellets having a pink to red appearance were formed upon centrifugation $(6,000 \mathrm{rpm}, 5 \mathrm{~min})$ and resuspended in $50 \mathrm{mM}$ Tris- $\mathrm{HCl}, \mathrm{pH}$. Cell lysing 
was performed through sonication, on ice, in the presence of cOmplete ${ }^{\mathrm{TM}}$, Mini, EDTA-free Protease Inhibitor Cocktail (Roche). Cellular debris was removed through centrifugation (13,000 rpm, $1 \mathrm{~h})$ affording a red transparent solution. The solution was purified on NiNTA resin and washed with 20 column volumes of $50 \mathrm{mM}$ Tris- $\mathrm{HCl}$ containing $10 \mathrm{mM}$ imidazole, pH 8. The protein was eluted with the same buffer containing $200 \mathrm{mM}$ imidazole. Imidazole was removed by repeated washing with $50 \mathrm{mM}$ Tris- $\mathrm{HCl}$, pH 8, buffer in 30,000 MW Amicon ${ }^{\circledR}$ Ultra-15 Centrifugal filters. Synthesis of $\left[\mathrm{Ru}(\mathrm{bpy})_{2}(5\right.$-iodoacetamido-1,10phenanthroline $)]\left(\mathrm{PF}_{6}\right)_{2}$ was performed as previously reported. ${ }^{40}$ Selective labeling of the triple and quadruple mutants at K97C was accomplished by mixing of 5 equivalents of photosensitizer with a $20 \mu \mathrm{M}$ solution of protein and shaken at $100 \mathrm{rpm}$ at $4{ }^{\circ} \mathrm{C}$ overnight. Excess photosensitizer was removed by centrifugal ultrafiltration and the labeled protein was concentrated and further purified by FPLC on a Mono Q anion exchange column to afford the RuP450 ${ }_{\mathrm{BM} 3}$ mutants. Labeling and sample purity were confirmed by UV-Vis and ESI-MS analysis.

\section{Transient Absorption Measurements}

Samples consisting of $10 \mu \mathrm{M}$ RuP450 ${ }_{\mathrm{BM} 3}$ mutant and oxidative quencher (20 mM ruthenium(III)hexaammine trichloride) were prepared in buffered solution ( $\mathrm{pH} \mathrm{8,50} \mathrm{mM} \mathrm{Tris-HCl).} \mathrm{Deoxy-}$ genation was achieved via 30 gentle pump-backfill cycles with argon while stirring. Samples were excited with $10 \mathrm{~ns}$ laser pulses at $460 \mathrm{~nm}$, delivered by an optical parametric oscillator pumped by the third harmonic from a Spectra Physics Q-switched Nd:YAG laser. Luminescence decays were monitored at $630 \mathrm{~nm}$. Single wavelength transient absorption (TA) kinetics were monitored at 390, 420, and $440 \mathrm{~nm}$ averaging $~ 500$ shots per wavelength. Data from four separate timescales $(2 \mu \mathrm{s}, 100 \mu \mathrm{s}, 10 \mathrm{~ms}$, and $500 \mathrm{~ms})$ were collected and spliced together to produce full kinetics traces. 


\section{Results and Discussion}

As Trp96 is the critical residue identified for electron hole transfer in P450 ${ }_{\mathrm{BM} 3},{ }^{7,12}$ our theoretical work has focused on the ET reaction displayed in Figure 4. We rely on available structural data from $\mathrm{P} 450_{2 \mathrm{~b} 4}$ to provide geometries of the Trp, Arg, and heme. ${ }^{19-23}$ We first examine an Arg to His mutation within Clusters A-D (Figure 5) to rationalize the effect on the absolute reduction potential of Trp. The choice of His was made to provide hydrogenbonding (H-bonding) to the heme propionate from the D pyrrole ring, similar to that seen in bacterial P450 enzymes. ${ }^{41,42}$ Next, we investigated how the distance of the Trp-Arg cation- $\pi$ interaction, and relative geometries of Trp-Arg-heme, perturb the absolute reduction potential of Trp and electronic coupling elements of Trp-heme, respectively. In this work, we selected a series of eight crystallographic structures from $\mathrm{P} 450_{2 \mathrm{~b} 4}$ and $\mathrm{P} 450_{\mathrm{BM} 3}$ that contain the analogous Trp-Arg pair and encompass the open, closed, and intermediate conformations of the dynamic enzyme (Figure 3).

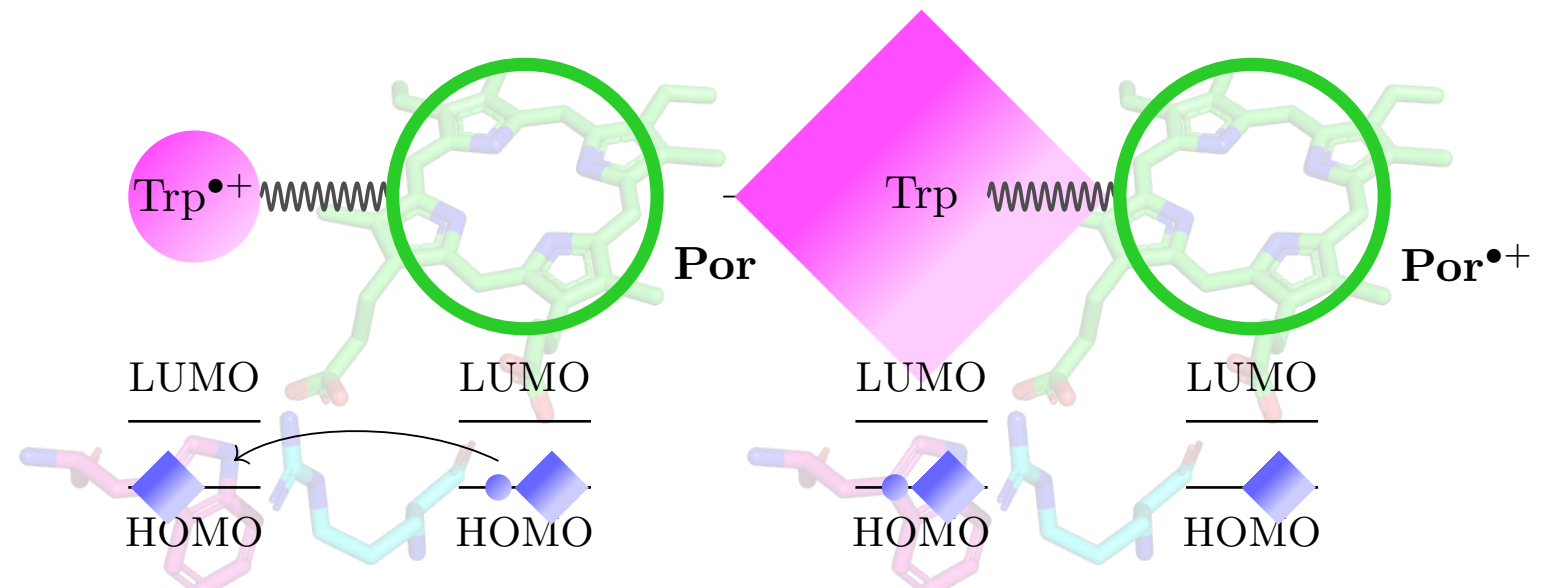

FIGURE. 4: A schematic view of the electron transfer step, for which we calculate the electronic coupling elements. An electron is transferred from the porphyrin ring of the heme to the tryptophan cation, $\operatorname{Trp}{ }^{\bullet+}$. 


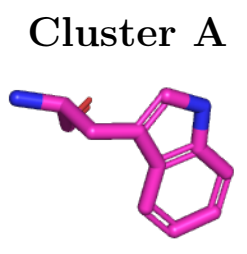

Cluster C
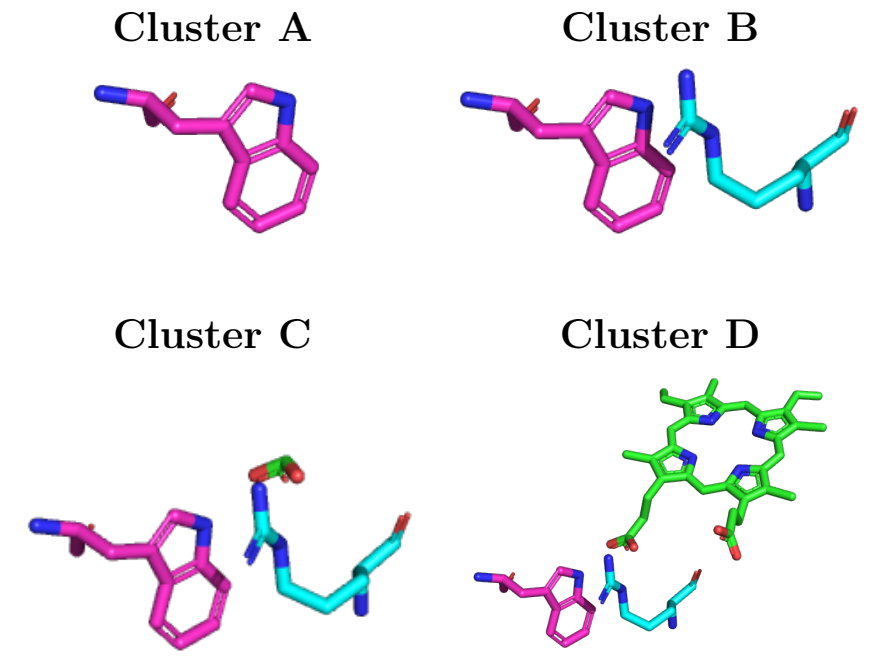

FIGURE. 5: Relative orientations of Trp residues (3NPL structure): (A) single Trp; (B) $\operatorname{Trp} / \operatorname{Arg}$ cluster; (C) $\operatorname{Trp} / \operatorname{Arg} /$ heme-substituent (acid group) cluster; and (D) $\operatorname{Trp} /$ Arg/heme cluster.

\section{Photooxidation Experiments}

We investigated the effect of the Arg398His mutation by single wavelength TA spectroscopy and time-resolved luminescence decay (Figure 6). The luminescence studies show nearly identical kinetics which support similar conformations of the Ru-photosensitizer and infer structural similarity of the two mutant enzymes. Moreover, prior crystallographic characterization shows that the Ru-label does not perturb protein structure. ${ }^{12}$ Taken together, we believe that the Arg398His mutant is structurally analogous to its non-mutant counterpart (likely due to a His-heme propionate H-bond).

We expect the Arg398His mutation, and loss of the cation- $\pi$ interaction, to lower the Trp96 reduction potential. Since Trp96 is essential in the photooxidation process and likely plays a role in protective hole transport from the heme, we anticipated a change in the transient absorption profiles and globally-fit kinetics (Figure 6). Our interpretation of the molecular processes corresponding to the TA changes are the same as previously reported. ${ }^{7,12}$ In this work, we fixed the first kinetics phase to the luminescence decay rate, furthermore, we added an additional kinetics phase in the slow time regime which afforded a slightly better global fit. Surprisingly, the single-wavelength TA traces are nearly identical in the wild type 

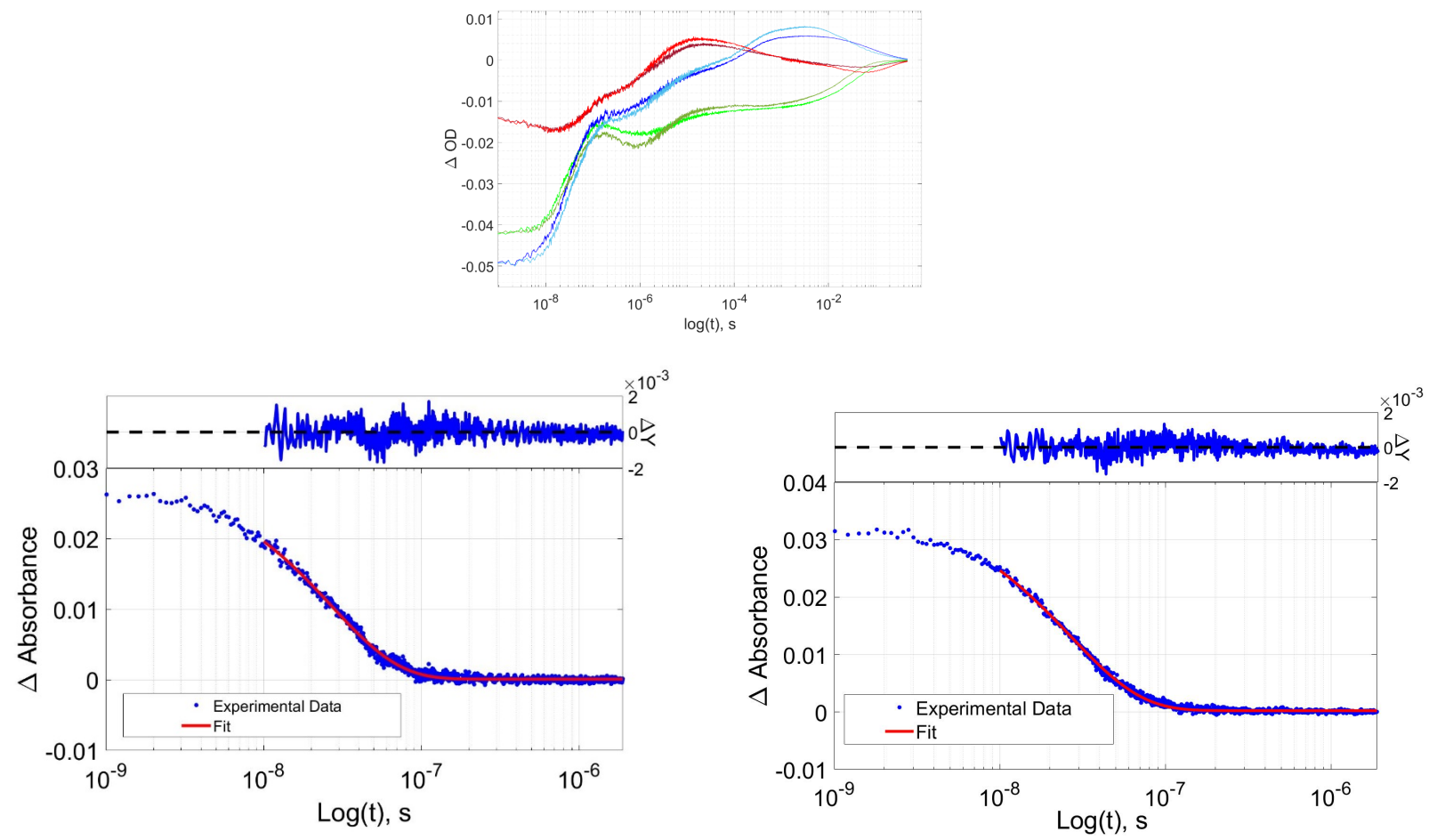

FIGURE. 6: (Top) TA kinetics traces for wild type and R398H RuP450 $0_{\mathrm{BM} 3}$ light red (R398H, $390 \mathrm{~nm}$ ), dark red (wt, $390 \mathrm{~nm}$ ), light green (R398H, $420 \mathrm{~nm}$ ), dark green (wt, $420 \mathrm{~nm}$ ), light blue (R398H, $440 \mathrm{~nm}$ ), dark blue (wt, $440 \mathrm{~nm}$ ). (Bottom) wild type RuP450 $0_{\mathrm{BM} 3}$ luminescence decay, (left); wild type RuP450 $0_{B M 3} \mathrm{R} 398 \mathrm{H}$ luminescence decay, (right). 
and Arg398His mutant. Global kinetics fits of each data set revealed similar kinetics for the six empirical kinetics phases (Table 1). Motivated by this intriguing result, we directed our efforts to a computational study of how conformational dynamics of the enzyme may control the reduction potential of a key Trp residue and the electronic coupling elements of the donor-acceptor pair.

Table 1: Rate constants from multi exponential global fitting of RuP450 TA kinetics. Phase 1 corresponds to the luminscence decay rate obtained from data in Figure 6.

\begin{tabular}{|c|c|c|}
\hline Kinetic Phase & Wild Type & R398H \\
\hline 1 & $3.7 \times 10^{7}$ & $3.8 \times 10^{7}$ \\
\hline 2 & $2.9 \times 10^{6}$ & $1.4 \times 10^{6}$ \\
\hline 3 & $2.2 \times 10^{5}$ & $2.0 \times 10^{5}$ \\
\hline 4 & $3.8 \times 10^{3}$ & $4.2 \times 10^{3}$ \\
\hline 5 & $4.8 \times 10^{1}$ & $3.6 \times 10^{1}$ \\
\hline 6 & $8.4 \times 10^{0}$ & $7.5 \times 10^{0}$ \\
\hline
\end{tabular}

\section{Calculation of the Reduction Potential}

In order to reduce computational time and maintain focus on the key Trp-Arg cation- $\pi$ interaction, we decided to study the four molecular Clusters shown in Figure 5. DFT calculations were performed on the Clusters in a dielectric medium which mimics the shielding of the surrounding protein (for a study of the solvation method see SI). This method cuts down on the computational costs while still allowing insight into the change in absolute reduction potential and electronic coupling elements.

Initially, we sought to understand how cluster size affects the absolute reduction potential of the wild type and Arg to His mutant enzymes. To accomplish this, we selected three crystal structures: 3NPL, 3R1A, and 3R1B. This data set offers two closed conformation structures (3NPL, P450 ${ }_{\mathrm{BM} 3}$ and $\left.3 \mathrm{R} 1 \mathrm{~A}, \mathrm{P} 450_{2 \mathrm{~b} 4}\right)$ in which the Trp-Arg cation- $\pi$ contact is present, and an intermediate structure where the $\operatorname{Trp}-\operatorname{Arg}$ cation- $\pi$ contact is lost while the Trp-heme distance is shortened $\left(3 \mathrm{R} 1 \mathrm{~B}, \mathrm{P} 450_{2 \mathrm{~b} 4}\right)$. The results of reduction potential as a function of 
Cluster size and geometry are summarized in Figure 7. For Cluster A, small variations in the reduction potential were calculated ranging from 1.06 to $1.19 \mathrm{~V}$ vs. NHE (SI Table 1), having good agreement with experimental values of Trp reduction potential of $1.015 \mathrm{eV}$ vs. NHE in aqueous solution at $\mathrm{pH} 7{ }^{43}$ Addition of the Arg to the Cluster shows an increase in the absolute reduction potential. We attribute this increase to a destabilization of $\operatorname{Trp}{ }^{\bullet+}$ by the cationic Arg residue. The calculated reduction potentials for Cluster B are higher than expected. However, the structural similarity in 3NPL and 3R1A afforded comparable reduction potentials, and the decrease in reduction potential of 3R1B, having a longer TrpArg distance, are encouraging. Across Clusters B-D, the replacement of the Arg cation with a neutral His residue showed an average decrease of $0.85 \mathrm{~V}$ vs NHE. We believe this value to be high, but still comparable to the experimentally measured increase of $0.35 \mathrm{~V}$ in a lysine (Lys)-Trp cation- $\pi$ interaction of a model protein. ${ }^{14}$

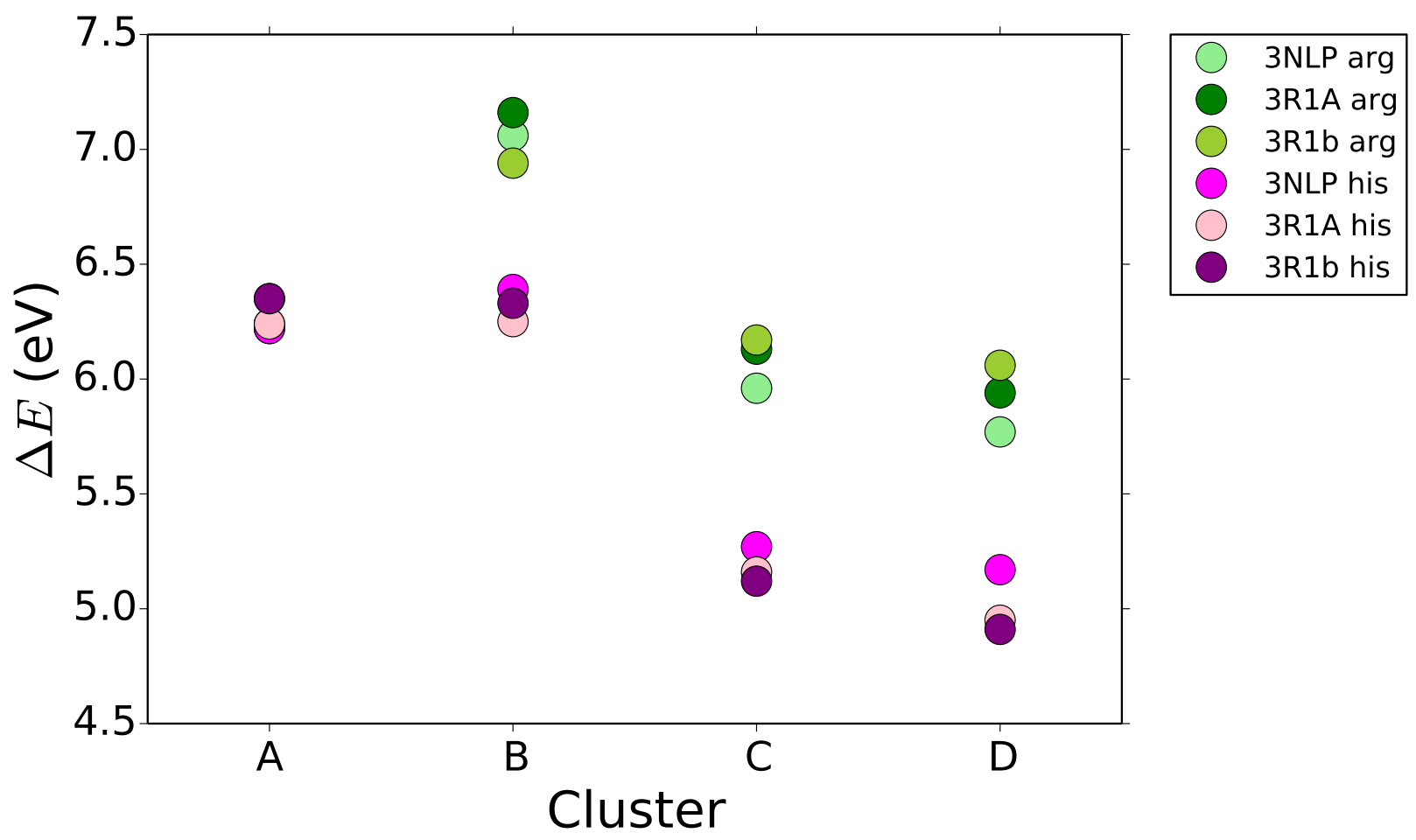

FIGURE. 7: Absolute reduction potential $(\Delta E)(\mathrm{eV})$ of each of the four cluster sizes, shown in Figure 5, for three different geometries of P450 both the wild type (in greens) and the His mutation (in pinks). Calculations performed with the PCM solvent model. 
When the anionic acid group was added to Clusters $\mathrm{C}$ and $\mathrm{D}$, the absolute reduction potential of the entire system decreased compared to Clusters A and B, Figure 7. We attribute the energy decrease to charge balance between the cationic Arg and anionic acid group, giving an absolute reduction potential slightly smaller than for just Trp. In the His Cluster the acid group helped to stabilize the cation in $\operatorname{Trp}^{\bullet+}$ giving a significantly lower absolute reduction potential. The combined effects of the Arg cation and the anionic acid demonstrate the sensitivity of Trp reduction potential to the surrounding motifs. Calculated reduction potentials depend highly on the Cluster size, yet the general trends upon introduction of Arg to His mutation, and the anionic acid component were similar among all Clusters. As none of these Clusters includes the full protein, we do not expect quantitative accuracy.

There are several factors which could contribute to the rate of ET in P450. Both the barrier height for the reaction and the electronic coupling elements between Trp and heme could play a role. The barrier height is affected by the absolute reduction potential of Trp. By mutating residues and changing the electrostatic surroundings, we anticipate modulation in the Trp absolute reduction potential. In many P450 enzymes, a Trp-Arg cation- $\pi$ interaction is present in close proximity to the heme, vide supra. This electrostatic interaction is expected to destabilize the one-electron oxidized $\operatorname{Trp}^{\bullet+}$, thereby raising the absolute reduction potential of Trp. By mutating the Arg to a neutral His, we expect the absolute reduction potential to decrease, thus decreasing the ET barrier height. In all cases where Arg/His are present we saw that cationic Arg destabilized Trp ${ }^{\bullet+}$ relative to the neutral His. Therefore, when Arg was mutated to His the absolute reduction potential decreased.

\section{Calculation of Electronic Coupling Elements}

We have studied the effect of mutation on the electronic coupling elements using four geometries, Table 2. The electronic coupling elements are calculated using two different methods, POD and FCD. The two methods were not in complete agreement, especially for the His mutations, owing to the choice of orbitals (see SI for more information). Generally, the 
wild type had smaller electronic coupling elements than the His mutants, except when the Trp-heme distance becomes large (as in the 1PO5 geometry) and then there is no electronic coupling calculated between the donor and acceptor. This result means that, when Arg is mutated to a His, the electronic coupling between Trp and heme becomes much stronger. Importantly, these results suggest that the Trp-Arg cation- $\pi$ interaction makes electron transfer less favorable in two ways, first by increasing the reduction potential of Trp, and second, by decreasing the electronic electronic coupling elements between Trp and heme.

Table 2: Electronic coupling elements (meV) between Trp and heme, calculated with both POD and FCD, in various P450 geometries which have different Trpheme distances $(\AA)$.

\begin{tabular}{|c|c|c|c|c|c|}
\hline \multirow{3}{*}{$\begin{array}{l}\text { Geometry } \\
\text { Cluster D }\end{array}$} & \multirow{3}{*}{$\begin{array}{c}\text { Distance }(\AA) \\
\text { Trp-heme }\end{array}$} & \multicolumn{4}{|c|}{ Coupling elements (meV) } \\
\hline & & \multicolumn{2}{|c|}{ Arg } & \multicolumn{2}{|c|}{ His } \\
\hline & & POD & FCD & POD & FCD \\
\hline $3 \mathrm{NPL}$ & 12.49 & 8.5 & 10.1 & 28 & 7.3 \\
\hline 3R1A & 12.50 & 3.7 & 2.7 & 73 & 33 \\
\hline 3R1B & 10.13 & 5.7 & 4.0 & 21 & 19 \\
\hline 1PO5 & 21.80 & 0.0 & 0.0 & 0.0 & 0.0 \\
\hline $3 \mathrm{G} 5 \mathrm{~N}$ & 12.19 & 11 & 10 & & \\
\hline 3G93 & 12.18 & 1.4 & 1.4 & & \\
\hline $2 \mathrm{BDM}$ & 16.32 & $3.6 \times 10^{-6}$ & $2.2 \times 10^{-4}$ & & \\
\hline 1DT6 & 13.07 & 11 & 12 & & \\
\hline
\end{tabular}

\section{Enzyme Conformational Dynamics and Reduction Potential/Electronic Coupling Elements}

The known structural dynamics of P450 enzymes led us to investigate more conformational geometries and their impact on reduction potential and electronic coupling elements. Specifically, we would like to know how the relative geometries between Trp-Arg and Trp-heme affect ET. Importantly, the photooxidation experiments were performed in solution at room temperature (RT). One must be cautious when applying crystal structure data to solution phenomena, however, the detailed work with $\mathrm{P} 450_{2 \mathrm{~b} 4}$ suggests that the extreme open confor- 
mation, 1PO5, exists in solution. ${ }^{19}$ Also, any protein mutation can cause unexpected structural changes. Notably, the luminescence decay data (Figure 6) indicate that solution structures of wild type and R398H have similar conformations (apparent in the single-exponential decay). Observation of multi-exponential decay would infer more than one conformation of the Ru-photosensitizer and therefore inconsistencies in the enzyme tertiary structure. In order to see what happens to the absolute reduction potential and the electronic coupling element when the Trp-Arg and Trp-heme distances change, we have studied computationally seven mammalian and one bacterial (3NPL) P450 conformations that have been determined by X-ray diffraction: 3G5N, 3G93, 2BDM, 3R1A, 1DT6, 3R1B, and 1PO5 (Figures 3 and $5)$.

\section{Absolute Reduction Potentials}

Using P450 geometries with varying Arg-Trp distances and relative orientations, we were able to study the geometric effects on the absolute reduction potential. These calculations were performed by using Clusters B-D where Arg is present. When only the Trp and Arg components are studied, Cluster B, we calculate a decrease in the absolute reduction potential as the Arg moves away from Trp. As anticipated, the further away the cationic Arg was from $\operatorname{Trp}^{\bullet+}$ the less it was destabilized, so the reduction potential was lowered. We can view this as a charge-charge interaction, meaning we would expect a $\frac{1}{r}$ decline, where $\mathrm{r}$ is the distance between Arg and Trp. We have fitted the data to a function of the form $\frac{a}{r}+b$ (seen as a pink line in Figure 8).

When the Cluster is expanded to include the acid group (Figure 8, Cluster C), the decrease with respect to distance disappeared (the data flattened out). In fact, the highest absolute reduction potential was found when Arg was furthest from the Trp. This finding can be understood by realizing that in 1PO5, which has the largest Trp-Arg distance, the influence of anything added near the Arg will have minimal influence on the Trp. The interaction between Trp and Arg-acid can be seen as a charge-dipole interaction, so we 


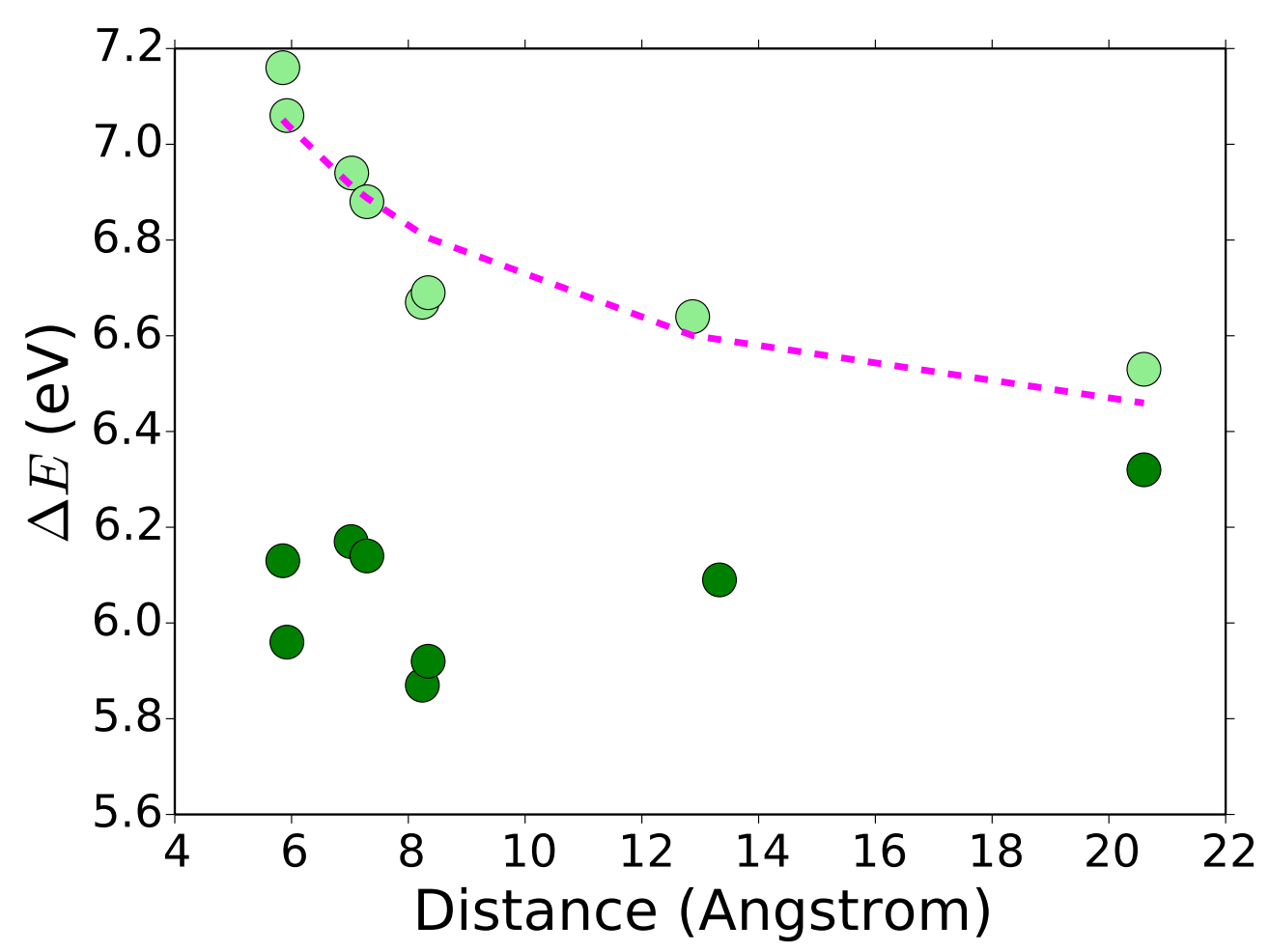

FIGURE. 8: Absolute reduction potential $(\Delta E)(\mathrm{eV})$, of (light green) Cluster B, and (dark green) Cluster $\mathrm{C}$, as shown in Figure 5. Various $\mathrm{P} 450$ geometries shown in relation to the Arg-Trp distance $(\AA)$. (Pink) Is a fit of a function of the form $\frac{a}{r}+b$ to the Cluster B absolute reduction potentials.

would expect a $\frac{1}{r^{2}}$ dependence. A $\frac{1}{r^{2}}$ dependence would be flatter than $\frac{1}{r}$ and we do see a flattening in Cluster C.

\section{Electronic Coupling Elements}

We calculated that changes in geometry and electrostatic environment have a large effect on the absolute reduction potential of Trp. Using two different methods (FCD and POD) we estimated electronic coupling parameters between Trp and heme for all the P450 geometries in Table 2. Here we did not see a strong distance to electronic coupling energy correlation when the Trp-heme distance was between 10-13 $\AA$. Note that we are working in very small energy units, meV, which means that small differences in geometry could have a large impact on the electronic coupling elements. Within 10-13 $\AA$ the large fluctuations could be due to differences in the geometries. We have investigated some of these differences including the 
angle between the $\pi$-system of Trp and the cation on Arg, but have found no clear correlation. However, when they move beyond $16 \AA$, the electronic coupling drops to almost zero, as would be expected.

\section{Conclusion}

We have studied the absolute reduction potential of Trp and the electronic coupling elements between Trp and heme in various wild type and mutated mammalian P450s. We used simple DFT calculations on Clusters of various sizes, geometries, and mutations in order to study the qualitative trends of absolute reduction potentials and electronic coupling elements. In this work, we found that the absolute reduction potential varies as the Cluster size increases. The absolute reduction potential is decreased when the cationic Arg in the wild type P450s is mutated to a neutral His. The distance between the Trp and the Arg can tune the absolute reduction potential in smaller Clusters. However, when an anionic acid is considered, the distance dependence is no longer as pronounced.

Interestingly, ET rates are unchanged when Arg398His $\mathrm{RuP} 450_{\mathrm{BM} 3}$ is analyzed by TA spectroscopy. This experimental observation is contrary to our calculations that show that variations in both the reduction potential and electronic coupling elements predict more rapid ET. Provided that Trp96 is essential to the photooxidation process, we conclude that there must be a conformational gating of the ET reaction. It follows that Trp96 must be in an intermediate conformation, away from Arg398, where the Arg398His mutation would have little to no effect on the Trp reduction potential, but maintain solution structure similarity.

Our working hypothesis is that when substrate is bound, the enzyme is in the closed position with a cation- $\pi$ interaction between Trp-Arg, thereby increasing the reduction potential of Trp and preventing this redox active amino acid from out competing substrate oxidation. When uncoupled turnover occurs, a conformational change would take place that separates the Trp-Arg pair, lowering the reduction potential to a more favorable level to 


\section{Supporting Information Description}

Supplementary information on computational details is provided.

\section{Acknowledgment}

Research reported in this publication was supported by the National Institute of Diabetes and Digestive and Kidney Diseases of the National Institutes of Health under award number R01DK019038. The content is solely the responsibility of the authors and does not necessarily represent the official views of the National Institutes of Health. T.H. thanks the Lundbeck Foundation for generous financial support. B.C.S. was supported by National Institute of Health under award number F32GM123639-01 and in part by U.S. Department of Energy 
(DOE), Oak Ridge National Laboratory Programmatic Fund during manuscript preparation.

\section{Author Information}

\section{Corresponding Author:}

E-mail: thorsten@chem.ku.dk

\section{Author Contribution}

MLHS and BCS contributed equally to this work. 


\section{References}

(1) Ortiz de Montellano, P. R. Hydrocarbon hydroxylation by cytochrome P450 enzymes. Chem. Rev. 2010, 110, 932-948.

(2) Rittle, J.; Younker, J. M.; Green, M. T. Cytochrome P450: The active oxidant and its spectrum. Inorg. Chem. 2010, 49, 3610-3617.

(3) Jung, S. T.; Lauchli, R.; Arnold, F. H. Cytochrome P450: taming a wild type enzyme. Curr. Opin. Biotech. 2011, 22, $809-817$.

(4) Nebert, D. W.; Wikvall, K.; Miller, W. L. Human cytochromes P450 in health and disease. Philos. T. R. Soc. B 2013, 368, 20120431.

(5) Urlacher, V. B.; Girhard, M. Cytochrome P450 monooxygenases in biotechnology and synthetic biology. Trends Biotechnol. 2019, 37, $882-897$.

(6) Sono, M.; Roach, M. P.; Coulter, E. D.; Dawson, J. H. Heme-containing oxygenases. Chem. Rev. 1996, 96, 2841-2888.

(7) Ener, M. E.; Gray, H. B.; Winkler, J. R. Hole hopping through tryptophan in cytochrome P450. Biochemistry 2017, 56, 3531-3538.

(8) Kathiresan, M.; English, A. M. LC-MS/MS proteoform profiling exposes cytochrome c peroxidase self-oxidation in mitochondria and functionally important hole hopping from its heme. J. Am. Chem. Soc. 2018, 140, 12033-12039.

(9) Kathiresan, M.; English, A. M. LC-MS/MS suggests that hole hopping in cytochrome c peroxidase protects its heme from oxidative modification by excess $\mathrm{H} 2 \mathrm{O}$ 2. Chem. Sci. 2017, 8, 1152-1162.

(10) Gray, H. B.; Winkler, J. R. Hole hopping through tyrosine/tryptophan chains protects proteins from oxidative damage. PNAS 2015, 112, 10920-10925. 
(11) Polizzi, N. F.; Migliore, A.; Therien, M. J.; Beratan, D. N. Defusing redox bombs? PNAS 2015, 112, 10821-10822.

(12) Ener, M. E.; Lee, Y.-T.; Winkler, J. R.; Gray, H. B.; Cheruzel, L. Photooxidation of cytochrome P450-BM3. PNAS 2010, 10\%, 18783-18786.

(13) Ma, J. C.; Dougherty, D. A. The cation- $\pi$ interaction. Chem. Rev. 1997, 97, 1303-1324.

(14) Tommos, C.; Skalicky, J. J.; Pilloud, D. L.; Wand, A. J.; Dutton, P. L. De novo proteins as models of radical enzymes. Biochemistry 1999, 38, 9495-9507.

(15) Dai, Q.-H.; Tommos, C.; Fuentes, E. J.; Blomberg, M. R.; Dutton, P. L.; Wand, A. J. Structure of a de novo designed protein model of radical enzymes. J. Am. Chem. Soc. 2002, 124, 10952-10953.

(16) Orabi, E. A.; English, A. M. Modeling protein S-aromatic motifs reveals their structural and redox flexibility. J. Phys. Chem. B 2018, 122, 3760-3770.

(17) Orabi, E. A.; English, A. M. Sulfur-aromatic interactions: Modeling cysteine and methionine binding to tyrosinate and histidinium ions to assess their influence on protein electron transfer. Isr. J. Chem. 2016, 56, 872-885.

(18) Genna, V.; Marcia, M.; De Vivo, M. A transient and flexible cation- $\pi$ interaction promotes hydrolysis of nucleic acids in DNA and RNA nucleases. J. Am. Chem. Soc. 2019,

(19) Scott, E. E.; He, Y. A.; Wester, M. R.; White, M. A.; Chin, C. C.; Halpert, J. R.; Johnson, E. F.; Stout, C. D. An open conformation of mammalian cytochrome P450 2B4 at 1.6-A resolution. PNAS 2003, 100, 13196-13201.

(20) Gay, S. C.; Zhang, H.; Wilderman, P. R.; Roberts, A. G.; Liu, T.; Li, S.; Lin, H.l.; Zhang, Q.; Woods Jr, V. L.; Stout, C. D. et al. Structural analysis of mammalian cytochrome P450 2B4 covalently bound to the mechanism-based inactivator 
tert-butylphenylacetylene: insight into partial enzymatic activity. Biochemistry 2011, 50, 4903-4911.

(21) Gay, S. C.; Sun, L.; Maekawa, K.; Halpert, J. R.; Stout, C. D. Crystal structures of cytochrome P450 2B4 in complex with the inhibitor 1-biphenyl-4-methyl-1 Himidazole: Ligand-induced structural response through $\alpha$-helical repositioning. Biochemistry 2009, 48, 4762-4771.

(22) Zhao, Y.; White, M. A.; Muralidhara, B.; Sun, L.; Halpert, J. R.; Stout, C. D. Structure of microsomal cytochrome P450 2B4 complexed with the antifungal drug bifonazole insight into P450 conformational plasticity and membrane interaction. J. Biol. Chem. 2006, 281, 5973-5981.

(23) Williams, P. A.; Cosme, J.; Sridhar, V.; Johnson, E. F.; McRee, D. E. Mammalian microsomal cytochrome P450 monooxygenase: structural adaptations for membrane binding and functional diversity. Mol. cell 2000, 5, 121-131.

(24) Frisch, M. J.; Trucks, G. W.; Schlegel, H. B.; Scuseria, G. E.; Robb, M. A.; Cheeseman, J. R.; Scalmani, G.; Barone, V.; Petersson, G. A.; Nakatsuji, H. et al. Gaussian 16 Revision A.03. 2016; Gaussian Inc. Wallingford CT.

(25) Zhao, Y.; Truhlar, D. G. The M06 suite of density functionals for main group thermochemistry, thermochemical kinetics, noncovalent interactions, excited states, and transition elements: two new functionals and systematic testing of four M06-class functionals and 12 other functionals. Theor. Chem. Acc. 2008, 120, 215-241.

(26) Hehre, W. J.; Ditchfield, R.; Pople, J. A. Self-consistent molecular orbital methods. XII. Further extensions of gaussian - type basis sets for use in molecular orbital studies of organic molecules. J. Chem. Phys. 1972, 56, 2257-2261.

(27) Francl, M. M.; Pietro, W. J.; Hehre, W. J.; Binkley, J. S.; Gordon, M. S.; DeFrees, D. J.; 
Pople, J. A. Self-consistent molecular orbital methods. XXIII. A polarization-type basis set for second-row elements. J. Chem. Phys. 1982, 77, 3654-3665.

(28) Frisch, M. J.; Pople, J. A.; Binkley, J. S. Self-consistent molecular orbital methods 25. Supplementary functions for gaussian basis sets. J. Chem. Phys. 1984, 80, 3265-3269.

(29) Clark, T.; Chandrasekhar, J.; Spitznagel, G. W.; Schleyer, P. V. R. Efficient diffuse function-augmented basis sets for anion calculations. III. The $3-21+\mathrm{G}$ basis set for first-row elements, Li-F. J. Comput. Chem. 1983, 4, 294-301.

(30) Tomasi, J.; Mennucci, B.; Cances, E. The IEF version of the PCM solvation method: an overview of a new method addressed to study molecular solutes at the QM ab initio level. J. Mol. Struc.: THEOCHEM 1999, 464, 211-226.

(31) Cossi, M.; Barone, V. Time-dependent density functional theory for molecules in liquid solutions. J. Chem. Phys. 2001, 115, 4708-4717.

(32) The PyMOL Molecular Graphics System, Version 2.0.7,Schrödinger, LLC.

(33) Hsu, C.-P. The electronic couplings in electron transfer and excitation energy transfer. Acc. Chem Res. 2009, 42, 509-518.

(34) Voityuk, A. A.; Rösch, N. Fragment charge difference method for estimating donoracceptor electronic coupling: Application to DNA $\pi$-stacks. J. Chem. Phys. 2002, 117, $5607-5616$.

(35) Mulliken, R. S. Electronic population analysis on LCAO-MO molecular wave functions. I. J. Chem. Phys. 1955, 23, 1833-1840.

(36) Kondov, I.; Čížek, M.; Benesch, C.; Wang, H.; Thoss, M. Quantum dynamics of photoinduced electron-transfer reactions in dye- semiconductor systems: First-principles description and application to coumarin 343- TiO2. J. Phys. Chem. C 2007, 111, $11970-11981$. 
(37) Futera, Z.; Blumberger, J. Electronic couplings for charge transfer across molecule/metal and molecule/semiconductor interfaces: Performance of the projector operator-based diabatization approach. J. Phys. Chem. C 2017, 121, 19677-19689.

(38) Löwdin, P. On the non-orthogonality problem connected with the use of atomic wave functions in the theory of molecules and crystals. J. Chem. Phys. 1950, 18, 365-375.

(39) Carlson, B. C.; Keller, J. M. Orthogonalization procedures and the localization of Wannier functions. Phys. Rev. 1957, 105, 102-103.

(40) Castellano, F. N.; Dattelbaum, J. D.; Lakowicz, J. R. Long-lifetime Ru (II) complexes as labeling reagents for sulfhydryl groups. Anal. Biochem. 1998, 255, 165-170.

(41) Poulos, T. L.; Finzel, B. C.; Howard, A. J. High-resolution crystal structure of cytochrome P450cam. J. Mol. Biol. 1987, 195, 687-700.

(42) Poulos, T. L.; Finzel, B. C.; Howard, A. J. High-resolution crystal structure of cytochrome P450cam. J. Mol. Biol. 1987, 195, 687-700.

(43) Harriman, A. Further comments on the redox potentials of tryptophan and tyrosine. J. Phys. Chem. 1987, 91, 6102-6104.

(44) Derbyshire, E. R.; Marletta, M. A. Structure and regulation of soluble guanylate cyclase. Annu. Rev. Biochem. 2012, 81, 533-559.

(45) Poulos, T. L. Heme enzyme structure and function. Chem. Rev. 2014, 114, 3919-3962.

(46) Sevrioukova, I. F.; Li, H.; Zhang, H.; Peterson, J. A.; Poulos, T. L. Structure of a cytochrome P450-redox partner electron-transfer complex. PNAS 1999, 96, 1863-1868.

(47) Batabyal, D.; Richards, L. S.; Poulos, T. L. Effect of redox partner binding on cytochrome P450 conformational dynamics. J. Am. Chem. Soc. 2017, 139, 13193-13199. 


\section{TOC Figure}

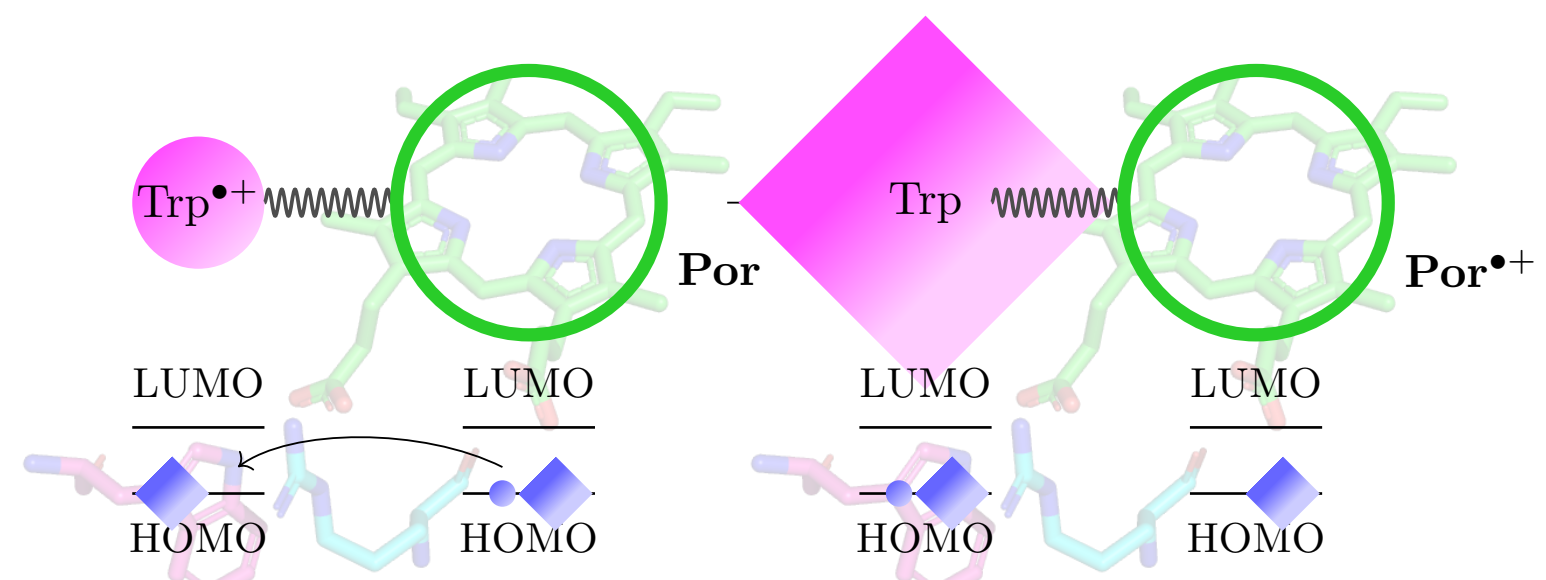




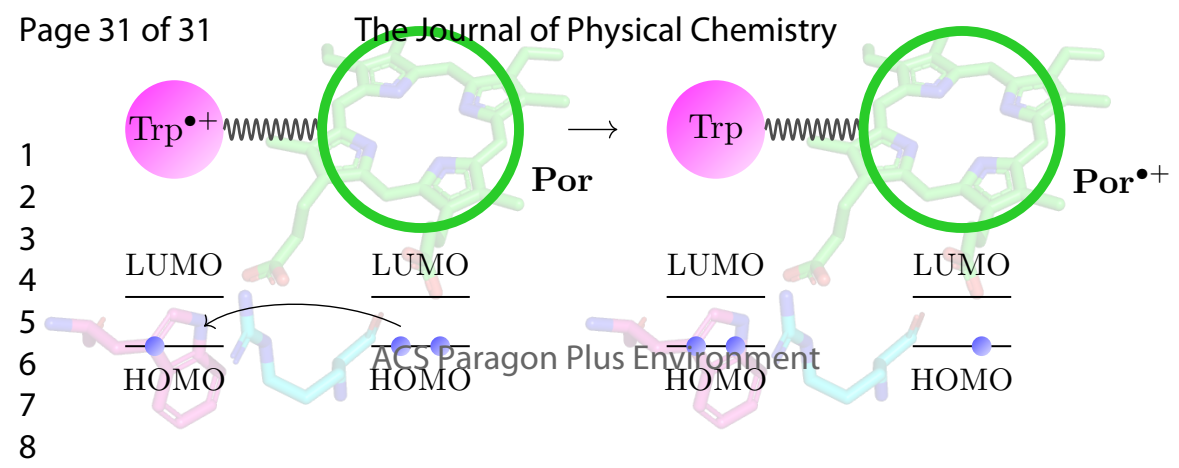

